\title{
EmbodimentExp
}

\section{Submission \#1119}

- 1 BoxPlot of all questionnaire items, groupment and overall embodiment (scaled to 5)

- 2 Embodiment questionnaire

- 2.1 Friedman and Wilcoxon tests for Overall Embodiment

- 2.2 Friedman and Wilcoxon tests for overall Ownership and particular ownership items

- 2.3 Friedman and Wilcoxon tests for overall Agency and particular agency items

- 2.4 Friedman and Wilcoxon tests for overall Tactile Sensations and particular tactile sensations items

- 2.5 Friedman and Wilcoxon tests for overall Self-Location and particular self-location items

- 3 Workload questionnaire

- 3.1 Friedman tests for NASA TLX items

- 3.2 Wilcoxon test for NASA TLX items

- 4 Performance over the drawings: Metrics analysis

- 4.1 Normality test over the Drawings metrics

- 4.2 ANOVA on ART for Completeness \& Accuracy

- 5 ANOVA On ART

- 5.1 Overall Embodiment

- 5.2 Groupment of Ownership

- 5.2.1 Item 01

- 5.2 .2 Item $\mathrm{O} 2$

- 5.2 .3 Item $\mathrm{O} 3$

- 5.3 Groupment of Agency

- 5.3.1 Item A1

- 5.3 .2 Item A2

- 5.3.3 Item A3

- 5.3.4 Item A4

- 5.4 Groupment of Self-Location

- 5.4.1 Item L1

- 5.4.2 Item L2

- 5.4 .3 Item L3

- 5.5 Groupment of Tactile sensations

- 5.5.1 Item T1

- 5.5.2 Item T2

- 5.5.3 Item T3

- 5.5.4 Post-hoc pairwise test for T3

- 5.5 .5 Item T4

- 5.6 ANOVA on ART for NASA TLX

- 5.6.1 Item Mental Load

- 5.6.2 Item Physical Load

- 5.6.3 Item Temporal Load

- 5.6.4 Item Performance

- 5.6 .5 Item Effort

- 5.6.6 Item Frustration

Loading data for analysis 


\section{BoxPlot of all questionnaire items, groupment and overall embodiment (scaled to 5)}

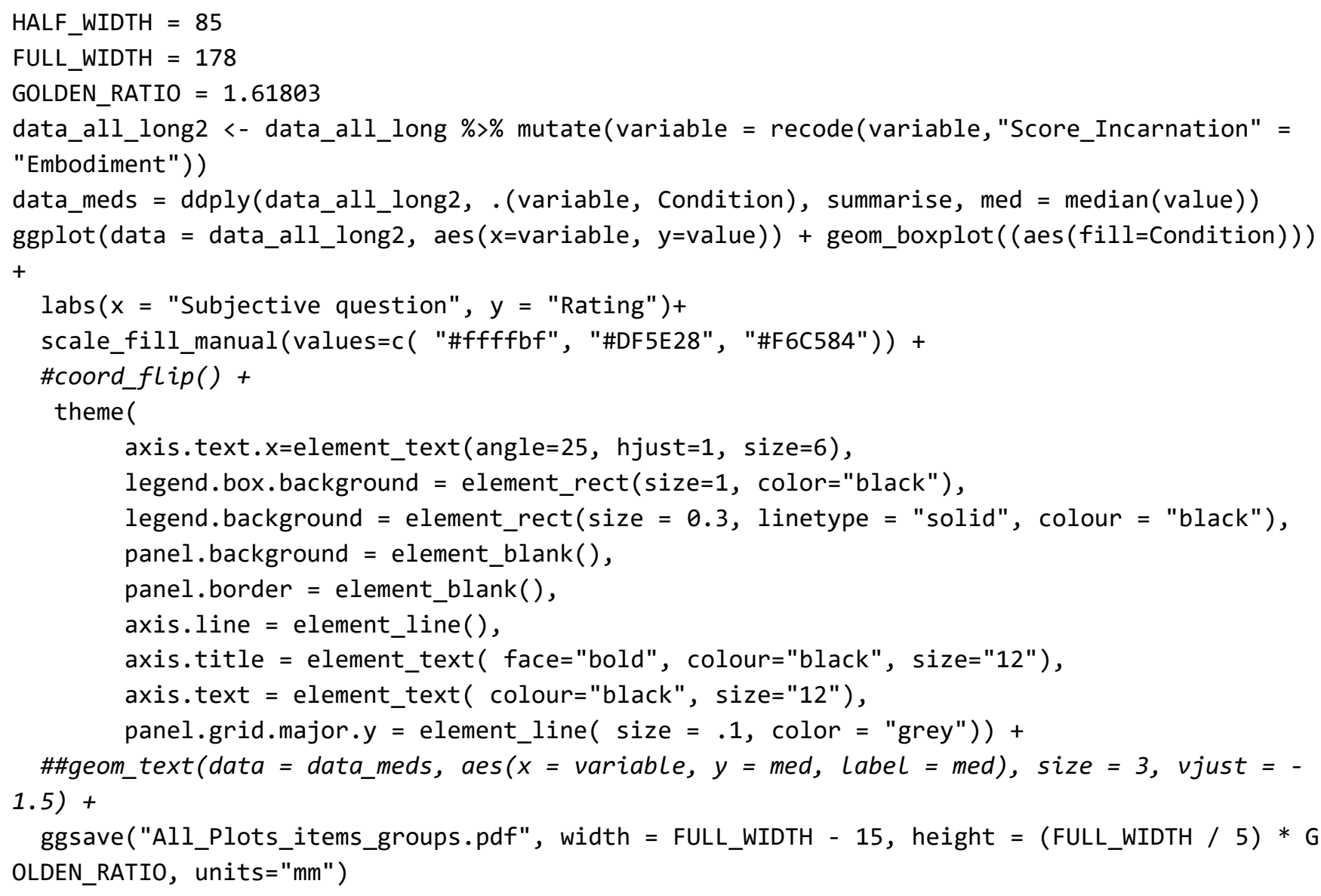




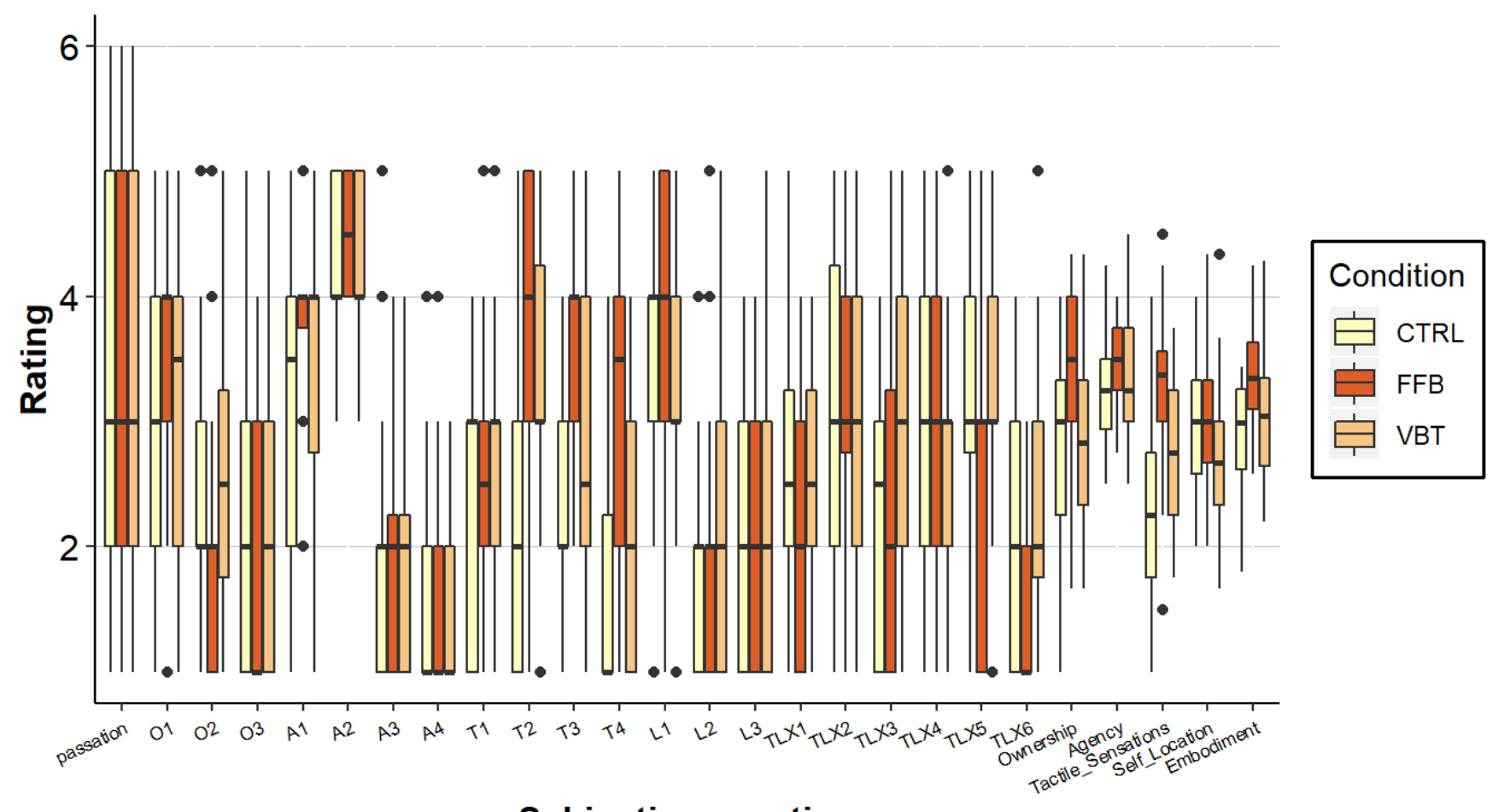

Subjective question

\section{Embodiment questionnaire}

\subsection{Friedman and Wilcoxon tests for Overall Embodiment}

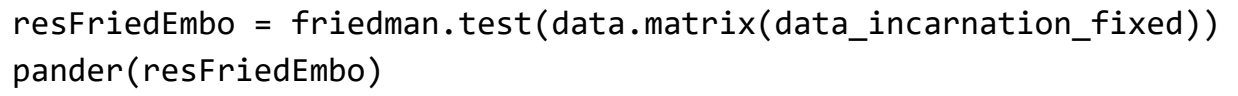

Friedman rank sum test:

data.matrix(data_incarnation_fixed)

\begin{tabular}{ccc} 
Test statistic & df & P value \\
\hline 52.05 & 3 & $2.916 \mathrm{e}-11 * * *$
\end{tabular}



CTRL 
resofried $=$ friedman.test $($ data. $\operatorname{matrix}($ data0 $))$

pander(resofried)

Friedman rank sum test: data.matrix(data0)

\begin{tabular}{ccc} 
Test statistic & df & P value \\
\hline 11.92 & 2 & $0.002581^{*}$
\end{tabular}

resOwil= pairwise. wilcox.test (dataOwil\$value, dataOwil\$condition, paired $=$ TRUE, $p \cdot$ adj $=$ "bo $n f ")$

kable(resOwil\$p.value)

CTRL.Ownership

FFB.Ownership

FFB.Ownership

0.0080828

1.0000000

VBT.Ownership

res01fried $=$ friedman.test $($ data $\cdot \operatorname{matrix}($ data01) $)$

pander (res01fried)

Friedman rank sum test: data.matrix(data01)

\begin{tabular}{ccc} 
Test statistic & df & P value \\
\hline 9.745 & 2 & $0.007652 *$ *
\end{tabular}

res01wil $=$ pairwise. wilcox.test $($ data01wil\$value, data01wil\$condition, paired $=$ TRUE, . $\cdot$ adj $=$ "bonf")

kable(res01wil\$p.value)

\section{CTRL.01}

0.0478279

0.5492902
FFB.01

FFB.01
VBT.01
$\begin{aligned} & \text { res02fried }=\text { friedman.test (data.matrix (data02) }) \\ & \text { pander (res02fried) }\end{aligned}$

FFB.O1

Friedman rank sum test: data.matrix(data02)

\begin{tabular}{ccc} 
Test statistic & df & P value \\
\hline 6.197 & 2 & 0.04512 *
\end{tabular}

res02wil $=$ pairwise. $w i l$ cox.test $($ data02wil\$value, data02wil\$condition, paired $=$ TRUE, $p \cdot$ adj $=$ "bonf")

kable(res02wil\$p.value) 
res03fried $=$ friedman. test $($ data $\cdot \operatorname{matrix}($ data03 $))$

pander (res03fried)

Friedman rank sum test: data.matrix(data03)

\begin{tabular}{ccc} 
Test statistic & df & P value \\
\hline 2.844 & 2 & 0.2412
\end{tabular}

\subsection{Friedman and Wilcoxon tests for overall Agency and particular agency items}

resAfried $=$ friedman.test $($ data $\cdot \operatorname{matrix}(\operatorname{dataA}))$

pander(resAfried)

Friedman rank sum test: data.matrix(dataA)

\begin{tabular}{ccc} 
Test statistic & df & P value \\
\hline 10.89 & 2 & $0.00432 * *$
\end{tabular}

resAwil $=$ pairwise $\cdot$ wilcox.test $($ dataAwil\$value, dataAwil\$condition, paired $=$ TRUE, $\mathrm{p} \cdot \operatorname{adj}=\mathrm{m}$ onf")

kable(resAwil\$p.value)

\section{CTRL.Agency}

0.1040757

0.5328913
FFB.Agency

FFB.Agency

VBT.Agency

resAlfried $=$ friedman.test $($ data $\cdot \operatorname{matrix}(\operatorname{dataA} 1))$

pander (resAlfried)

Friedman rank sum test: data.matrix(dataA1)

\begin{tabular}{ccc} 
Test statistic & df & P value \\
\hline 11.66 & 2 & $0.002936^{*}$ *
\end{tabular}

resAlwil $=$ pairwise. wilcox.test $($ dataA1wil\$value, dataA1wil\$condition, paired $=$ TRUE, $p \cdot \operatorname{adj}=$ "bonf")

kable(resA1wil\$p.value) 
resA2fried $=$ friedman.test $($ data $\cdot \operatorname{matrix}(\operatorname{dataA2}))$

pander(resA2fried)

Friedman rank sum test: data.matrix(dataA2)

\begin{tabular}{ccc} 
Test statistic & df & P value \\
\hline 2 & 2 & 0.3679
\end{tabular}

resA3fried $=$ friedman.test $($ data. $\operatorname{matrix}($ dataA 3$)$ )

pander (resA3fried)

Friedman rank sum test: data.matrix(dataA3)

$\begin{array}{ccc}\text { Test statistic } & \text { df } & \text { P value } \\ 0.1404 & 2 & 0.9322\end{array}$

resA4fried $=$ friedman.test $($ data. $\operatorname{matrix}($ dataA4 $))$

pander (resA4fried)

Friedman rank sum test: data.matrix(dataA4)

\begin{tabular}{ccc} 
Test statistic & df & P value \\
\hline 0.5625 & 2 & 0.7548
\end{tabular}

\subsection{Friedman and Wilcoxon tests for overall Tactile Sensations and particular tactile sensations items}

resTfried $=$ friedman.test $($ data $\cdot \operatorname{matrix}($ dataT $))$

pander(resTfried)

Friedman rank sum test: data.matrix(dataT)

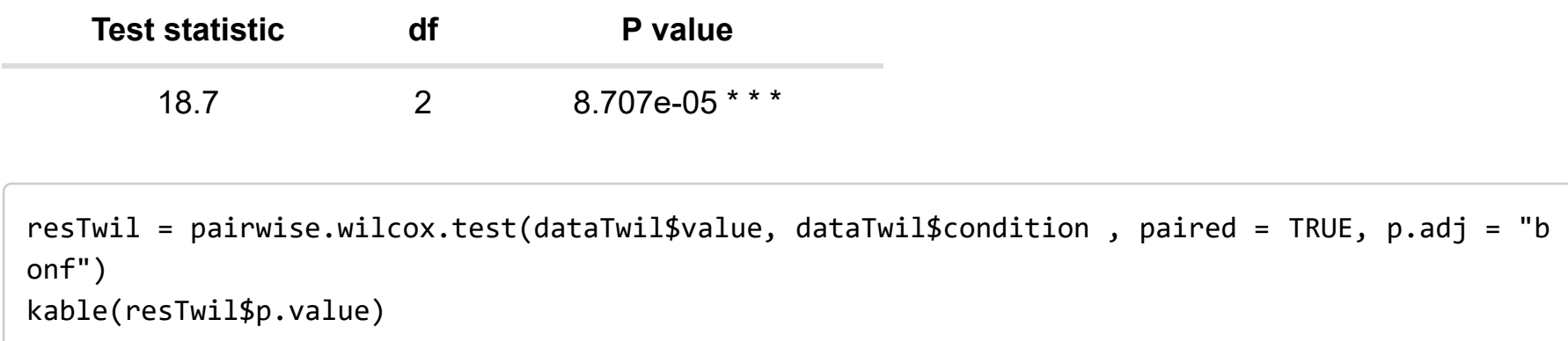




FFB.Tactile_Sensations
VBT.Tactile_Sensations
$\begin{aligned} & \text { resT1fried }=\text { friedman.test (data.matrix (dataT1)) } \\ & \text { pander(resT1fried) }\end{aligned}$

Friedman rank sum test: data.matrix(dataT1)

\begin{tabular}{ccc} 
Test statistic & df & P value \\
\hline 0.3939 & 2 & 0.8212
\end{tabular}

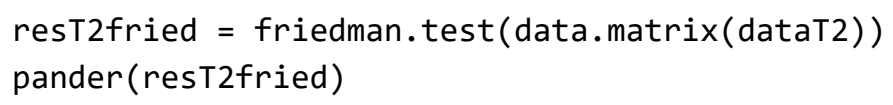

Friedman rank sum test: data.matrix(dataT2)

\begin{tabular}{ccc} 
Test statistic & df & P value \\
\hline 14.14 & 2 & $0.0008515 * * *$
\end{tabular}

resT2wil = pairwise.wilcox.test(dataT2wil\$value, dataT 2 wil\$condition, paired = TRUE, $\mathrm{p}$. adj $=$ "bonf")

kable(rest2wil\$p.value)

CTRL.T2

FFB.T2

FFB.T2

0.0063507

0.0099834

VBT.T2

resT3fried $=$ friedman.test $($ data $\cdot \operatorname{matrix}($ dataT3 $))$

pander(rest3fried)

Friedman rank sum test: data.matrix(dataT3)

\begin{tabular}{ccc} 
Test statistic & df & P value \\
\hline 16.12 & 2 & $0.0003157 * * *$
\end{tabular}

resT3wil = pairwise. wilcox.test (dataT3wil\$value, dataT3wil\$condition, paired = TRUE, $\mathrm{p}$. adj $=$ "bonf")

kable (rest3wil\$p.value) 
resT4fried $=$ friedman.test $($ data $\cdot \operatorname{matrix}($ dataT4 $))$

pander(rest4fried)

Friedman rank sum test: data.matrix(dataT4)

\begin{tabular}{ccc} 
Test statistic & df & P value \\
\hline 11.25 & 2 & $0.003613^{*}$
\end{tabular}

resT4wil = pairwise.wilcox.test (dataT4wil\$value, dataT4wil\$condition, paired = TRUE, $\mathrm{p}$. adj $=$ "bonf")

kable (resT4wil\$p.value)

CTRL.T4

FFB.T4

FFB.T4

0.0055365

NA

VBT.T4

0.3192144

0.010308

\subsection{Friedman and Wilcoxon tests for overall Self- Location and particular self-location items}

res $L=$ friedman.test $($ data $\cdot \operatorname{matrix}($ data $L))$

pander (resL)

Friedman rank sum test: data.matrix(dataL)

\begin{tabular}{ccc} 
Test statistic & df & P value \\
\hline 4.083 & 2 & 0.1298
\end{tabular}

resL1fried = friedman.test $($ data $\cdot \operatorname{matrix}($ dataL1) $)$

pander(resL1fried)

Friedman rank sum test: data.matrix(dataL1)

\begin{tabular}{ccc} 
Test statistic & df & P value \\
\hline 5.045 & 2 & 0.08027
\end{tabular}

resL2fried $=$ friedman.test $($ data. $\operatorname{matrix}($ dataL 2$))$

pander(resL2fried)

Friedman rank sum test: data.matrix(dataL2)

\begin{tabular}{ccc} 
Test statistic & df & P value \\
\hline 2.627 & 2 & 0.2688
\end{tabular}


resL3fried $=$ friedman.test $($ data $\cdot \operatorname{matrix}($ dataL3) $)$

pander(resL3fried)

Friedman rank sum test: data.matrix(dataL3)

\begin{tabular}{ccc} 
Test statistic & df & P value \\
\hline 0.16 & 2 & 0.9231
\end{tabular}

\section{Workload questionnaire}

\subsection{Friedman tests for NASA TLX items}

restlx1 = friedman.test $($ data. $\operatorname{matrix}($ dataTLX_Mental $))$

pander(restlx1)

Friedman rank sum test:

data.matrix(dataTLX_Mental)

\begin{tabular}{ccc} 
Test statistic & df & P value \\
\hline 7.284 & 2 & $0.02621^{*}$
\end{tabular}

restlx2 = friedman.test(data.matrix(dataTLX_Physical))

pander (restlx2)

Friedman rank sum test:

data.matrix(dataTLX_Physical)

\begin{tabular}{ccc} 
Test statistic & df & P value \\
\hline 2.259 & 2 & 0.3232
\end{tabular}

restlx3 = friedman.test (data.matrix (dataTLX_Temporal) $)$

pander(restlx3)

Friedman rank sum test:

data.matrix(dataTLX_Temporal)

\begin{tabular}{ccc} 
Test statistic & df & P value \\
\hline 9.234 & 2 & $0.009882 * *$
\end{tabular}

restlx4 = friedman.test (data.matrix (dataTLX_Performance) $)$

pander (restlx4)

Friedman rank sum test:

data.matrix(dataTLX_Performance) 


\begin{tabular}{ccc} 
Test statistic & df & P value \\
\hline 9.508 & 2 & $0.008618^{* *}$ \\
\hline $\begin{array}{l}\text { restl } \times 5=\text { friedman.test(data.matrix(dataTLX_Effort)) } \\
\text { pander(restl } \times 5)\end{array}$
\end{tabular}

Friedman rank sum test: data.matrix(dataTLX_Effort)

\begin{tabular}{ccc} 
Test statistic & df & P value \\
\hline 9.524 & 2 & $0.008549^{*}$ *
\end{tabular}

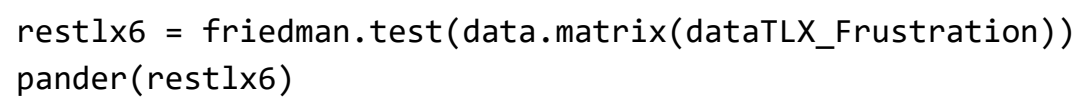

Friedman rank sum test:

data.matrix(dataTLX_Frustration)

\begin{tabular}{ccc} 
Test statistic & df & P value \\
\hline 10.75 & 2 & $0.004631 * *$
\end{tabular}

\subsection{Wilcoxon test for NASA TLX items}

resTLX1 = pairwise.wilcox.test(dataTLX_Mentalwil\$value, dataTLX_Mentalwil\$condition, paired $=$ TRUE, $p \cdot$ adj $=$ "bonf")

kable(resTLX1\$p.value)

\section{CTRL.TLX1}

0.0490675

1.0000000
FFB.TLX1

NA

0.034517

VBT.TLX1

CTRL.TLX2

FFB.TLX2

resTLX2 = pairwise.wilcox.test(dataTLX_Physicalwil\$Value, dataTLX_Physicalwil\$condition, pai red $=$ TRUE, p.adj = "bonf")

kable(resTLX2\$p.value)

FFB.TLX2

1

NA

VBT.TLX2

1

1

resTLX3 = pairwise.wilcox.test(dataTLX_Temporalwil\$value, dataTLX_Temporalwil\$condition , pai red $=$ TRUE, . adj = "bonf")

kable(resTLX3\$p.value) 
resTLX4 = pairwise.wilcox.test(dataTLX_Performancewil\$value, dataTLX_Performancewil\$condition , paired = TRUE, $\mathrm{p} \cdot \mathrm{adj}=$ "bonf")

kable(resTLX4\$p.value)

\section{CTRL.TLX4}

FFB.TLX4

FFB.TLX4

1.0000000

NA

VBT.TLX4

0.1484883

0.0148417

resTLX5 = pairwise.wilcox.test(dataTLX_Effortwil\$value, dataTLX_Effortwil\$condition , paired

$=$ TRUE, p.adj = "bonf")

kable(resTLX5\$p.value)

CTRL.TLX5

FFB.TLX5

FFB.TLX5

0.0231635

NA

VBT.TLX5

1.0000000

0.0308577

resTLX6 = pairwise.wilcox.test(dataTLX_Frustrationwil\$value, dataTLX_Frustrationwil\$condition , paired = TRUE, $p \cdot$ adj = "bonf")

kable(resTLX6\$p.value)

CTRL.TLX6

FFB.TLX6

FFB.TLX6

0.0490675

NA

VBT.TLX6

1.0000000

0.0103015

\section{Performance over the drawings: Metrics analysis}

\subsection{Normality test over the Drawings metrics}

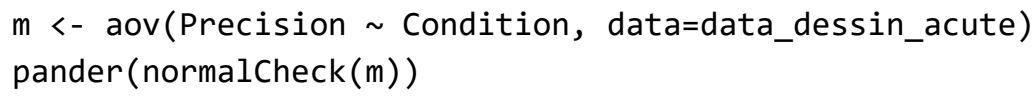

Quitting from lines 379-384 (EmbodimentExpResults_plot.Rmd) Error in normalCheck $(\mathrm{m})$ : impossible de trouver la fonction "normalCheck" Calls: ... withCallingHandlers -> withVisible -> eval -> eval -> pander De plus : There were 50 or more warnings (use warnings() to see the first 50 ) 


\subsection{ANOVA on ART for Completeness \& Accuracy}

mCompl $=\operatorname{art}($ Completeness $\sim$ factor(Condition $)+(1 \mid$ participant $)$, data $=$ data_dessin_compl $)$ kable(anova (mCompl))

\begin{tabular}{llrrrr} 
& Term & F & Df & Df.res & $\operatorname{Pr}(>$ F $)$ \\
\hline factor(Condition) & factor(Condition) & 1.353551 & 2 & 46 & 0.2684153
\end{tabular}

mAcute $=\operatorname{art}($ Precision $\sim$ factor(Condition $)+(1 \mid$ participant $)$, data $=$ data_dessin_acute $)$ kable(anova(mAcute))

\begin{tabular}{llrrrr} 
& Term & F & Df & Df.res & $\operatorname{Pr}(>$ F) \\
\hline factor(Condition) & factor(Condition) & 1.325216 & 2 & 46 & 0.2756995
\end{tabular}

\section{ANOVA on ART}

\subsection{Overall Embodiment}

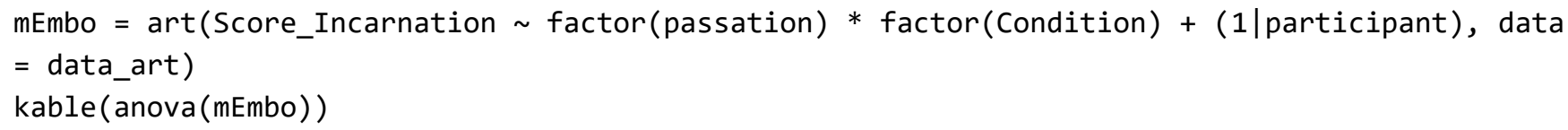

Term

factor(passation)

factor(Condition)

factor(Condition)

factor(passation):factor(Condition) factor(passation):factor(Condition)

F Df Df.res

$\operatorname{Pr}(>\mathrm{F})$

\begin{tabular}{|c|c|c|c|c|c|}
\hline factor(passation) & factor(passation) & 2.1191420 & 5 & 18 & 0.1099136 \\
\hline factor(Condition) & factor(Condition) & 9.3460131 & 2 & 36 & 0.0005380 \\
\hline factor(passation):factor(Co & factor(passation):factor(Condition) & 0.9099813 & 10 & 36 & 0.534492 \\
\hline
\end{tabular}

\subsection{Groupment of Ownership}

m0 = art(Ownership $~$ factor(passation) $*$ factor (Condition) $+(1 \mid$ participant), data = data_ar t) kable(anova $(\mathrm{mo}))$

Term

factor(passation)

factor(Condition)

factor(passation):factor(Condition) factor(passation):factor(Condition) factor(passation)

factor(Condition)
F Df Df.res

$\operatorname{Pr}(>F)$

$\begin{array}{rrrr}1.3080741 & 5 & 18 & 0.3044646 \\ 5.2446392 & 2 & 36 & 0.0100252 \\ 0.8788101 & 10 & 36 & 0.5608743\end{array}$


m01 = art(01 factor (passation) * factor(Condition) + (1|participant), data = data1)

kable(anova (m01))

\section{Term}

factor(passation)

factor(Condition)

factor(passation):factor(Condition) factor(passation):factor(Condition)

factor(passation)
F Df Df.res

$\operatorname{Pr}(>\mathrm{F})$

$\begin{array}{llll}2.055169 & 5 & 18 & 0.1189762\end{array}$

$\begin{array}{llll}5.638673 & 2 & 36 & 0.0074078\end{array}$

$\begin{array}{llll}1.000878 & 10 & 36 & 0.4610882\end{array}$

\section{2 .2 Item $\mathrm{O} 2$}

m02 $=\operatorname{art}(02 \sim$ factor $($ passation $) *$ factor $($ Condition $)+(1 \mid$ participant $)$, data $=$ data 1$)$

kable(anova $(\mathrm{mO2}))$

Term

\begin{tabular}{llrrrr}
\hline factor(passation) & factor(passation) & 1.8729749 & 5 & 18 & 0.1493399 \\
\hline factor(Condition) & factor(Condition) & 3.3085841 & 2 & 36 & 0.0479648 \\
\hline factor(passation):factor(Condition) & factor(passation):factor(Condition) & 0.4232267 & 10 & 36 & 0.9258305
\end{tabular}

\section{2 .3 Item $\mathrm{O} 3$}

m03 $=\operatorname{art}(03 \sim$ factor $($ passation $) *$ factor $($ Condition $)+(1 \mid$ participant $)$, data $=$ data 1$)$

$\operatorname{kable}(\operatorname{anova}(\mathrm{mO3}))$

\section{Term}

F Df Df.res

$\operatorname{Pr}(>\mathrm{F})$

\begin{tabular}{llllll}
\hline factor(passation) & factor(passation) & 0.6447526 & 5 & 18 & 0.6689026 \\
\hline factor(Condition) & factor(Condition) & 0.6298155 & 2 & 36 & 0.5384571 \\
\hline factor(passation):factor(Condition) & factor(passation):factor(Condition) & 0.9981044 & 10 & 36 & 0.4632400
\end{tabular}

\subsection{Groupment of Agency}

$\mathrm{mA}=\operatorname{art}($ Agency $\sim$ factor $($ passation $) *$ factor(Condition $)+(1 \mid$ participant $)$, data $=$ data_art $)$ $\operatorname{kable}(\operatorname{anova}(\mathrm{mA}))$

Term

factor(passation)

factor(Condition)

factor(passation):factor(Condition) factor(passation)

factor(Condition)

factor(passation):factor(Condition)

\section{F Df Df.res}

$\begin{array}{llll}1.152550 \quad 5 & 18 & 0.3697127\end{array}$

$\begin{array}{llll}3.433788 & 2 & 36 & 0.0431643\end{array}$

1.07826410

$36 \quad 0.4035621$

\subsubsection{Item A1}


$\mathrm{mA1}=\operatorname{art}(\mathrm{A} 1 \sim$ factor $($ passation $) *$ factor(Condition $)+(1 \mid$ participant $)$, data $=$ data1 $)$

kable(anova(mA1))

\section{Term}

factor(passation)

factor(Condition)

factor(Condition)

factor(passation):factor(Condition) factor(passation):factor(Condition)

F Df Df.res

$\operatorname{Pr}(>\mathrm{F})$

\begin{tabular}{llllll} 
factor(passation) & factor(passation) & 1.045195 & 5 & 18 & 0.4218926 \\
\hline factor(Condition) & factor(Condition) & 6.068108 & 2 & 36 & 0.0053573 \\
\hline factor(passation):factor(Condition) & factor(passation):factor(Condition) & 1.125009 & 10 & 36 & 0.3712721
\end{tabular}

\subsubsection{Item A2}

$\mathrm{mA} 2=\operatorname{art}(\mathrm{A} 2 \sim$ factor $($ passation $) *$ factor(Condition $)+(1 \mid$ participant $)$, data $=$ data1 $)$

kable(anova (mA2))

Term

factor(passation)

factor(Condition)

factor(Condition)

factor(passation):factor(Condition) factor(passation):factor(Condition)

F Df Df.res

$\operatorname{Pr}(>\mathrm{F})$

\begin{tabular}{llllll} 
factor(passation) & factor(passation) & 0.2908477 & 5 & 18 & 0.9118236 \\
\hline factor(Condition) & factor(Condition) & 1.8615571 & 2 & 36 & 0.1700849 \\
\hline factor(passation):factor(Condition) & factor(passation):factor(Condition) & 4.4155410 & 10 & 36 & 0.0004448
\end{tabular}

\subsubsection{Item A3}

$\mathrm{mA3}=\operatorname{art}(\mathrm{A} 3 \sim$ factor $($ passation $) *$ factor(Condition $)+(1 \mid$ participant $)$, data $=$ data 1$)$

kable(anova (mA3))

\section{Term}

factor(passation)

factor(Condition)

factor(Condition)

factor(passation):factor(Condition) factor(passation):factor(Condition)

F Df Df.res

$\operatorname{Pr}(>\mathrm{F})$

\begin{tabular}{llllll}
\hline factor(passation) & factor(passation) & 0.8824209 & 5 & 18 & 0.5127435 \\
\hline factor(Condition) & factor(Condition) & 0.1010823 & 2 & 36 & 0.9041142 \\
\hline factor(passation):factor(Condition) & factor(passation):factor(Condition) & 1.0017260 & 10 & 36 & 0.4604317
\end{tabular}

\subsubsection{Item A4}

$\mathrm{mA} 4=\operatorname{art}(\mathrm{A} 4 \sim$ factor $($ passation $) *$ factor(Condition $)+(1 \mid$ participant $)$, data $=$ data 1$)$

kable(anova (mA4))

\section{Term}

factor(passation)

factor(Condition)

factor(passation):factor(Condition) factor(passation):factor(Condition)

factor(passation)
F Df Df.res

$\operatorname{Pr}(>\mathrm{F})$

$\begin{array}{llll}1.0711703 & 5 & 18 & 0.4087097\end{array}$

$\begin{array}{llll}0.6527931 & 2 & 36 & 0.5266419\end{array}$

$\begin{array}{llll}0.7074195 & 10 & 36 & 0.7114518\end{array}$

\subsection{Groupment of Self-Location}


$\mathrm{mSL}=\operatorname{art}($ Self_Location $\sim$ factor $($ passation $) *$ factor $($ Condition $)+(1 \mid$ participant $)$, data = dat a_art)

$\operatorname{kable}(\operatorname{anova}(m S L))$

Term

factor(passation)

factor(passation)

factor(Condition)

factor(passation):factor(Condition) factor(passation):factor(Condition)
F Df Df.res

$\operatorname{Pr}(>\mathrm{F})$

$\begin{array}{rrrr}1.501649 & 5 & 18 & 0.2385168 \\ 1.607882 & 2 & 36 & 0.2143644 \\ 1.279717 & 10 & 36 & 0.2781018\end{array}$

\subsubsection{Item L1}

$\mathrm{mL1}=\operatorname{art}(\mathrm{L1} \sim$ factor(passation) $*$ factor(Condition) $+(1 \mid$ participant $)$, data $=$ data1 $)$ kable(anova $(m L 1))$

Term

\begin{tabular}{llrrrr}
\hline factor(passation) & factor(passation) & 2.5224346 & 5 & 18 & 0.0672680 \\
\hline factor(Condition) & factor(Condition) & 2.1379655 & 2 & 36 & 0.1326247 \\
\hline factor(passation):factor(Condition) & factor(passation):factor(Condition) & 0.9843207 & 10 & 36 & 0.4740202
\end{tabular}

\subsubsection{Item L2}

$\mathrm{mL2}=\operatorname{art}(\mathrm{L2} \sim$ factor (passation) $*$ factor(Condition) $+(1 \mid$ participant $)$, data $=$ data1 $)$ kable $($ anova $(\mathrm{mL} 2))$

\section{Term}

factor(passation)

factor(Condition)

factor(Condition)

factor(passation):factor(Condition) factor(passation):factor(Condition)

F Df Df.res

$\operatorname{Pr}(>\mathrm{F})$

\begin{tabular}{llrrrr}
\hline factor(passation) & factor(passation) & 0.7402051 & 5 & 18 & 0.6032919 \\
\hline factor(Condition) & factor(Condition) & 0.2678939 & 2 & 36 & 0.7665005 \\
\hline factor(passation):factor(Condition) & factor(passation):factor(Condition) & 1.3236833 & 10 & 36 & 0.2553947
\end{tabular}

\subsubsection{Item L3}

$\mathrm{mL} 3=\operatorname{art}(\mathrm{L} 3 \sim$ factor(passation) $*$ factor(Condition $)+(1 \mid$ participant $)$, data $=$ data1 $)$ $\operatorname{kable}(\operatorname{anova}(\mathrm{mL} 3))$

\section{Term}

factor(passation)

factor(Condition)

factor(passation):factor(Condition) factor(passation):factor(Condition)

\section{F Df Df.res}

$\begin{array}{rrrr}0.3318429 & 5 & 18 & 0.8870724 \\ 0.2044999 & 2 & 36 & 0.8159951 \\ 1.8404970 & 10 & 36 & 0.0882412\end{array}$

\subsection{Groupment of Tactile sensations}






Term

F Df Df.res

$\operatorname{Pr}(>\mathrm{F})$

\begin{tabular}{llrrrr}
\hline factor(passation) & factor(passation) & 2.3631066 & 5 & 18 & 0.0815170 \\
\hline factor(Condition) & factor(Condition) & 14.6441560 & 2 & 36 & 0.0000222 \\
\hline factor(passation):factor(Condition) & factor(passation):factor(Condition) & 0.7787407 & 10 & 36 & 0.6482996
\end{tabular}

\subsubsection{Item T1}

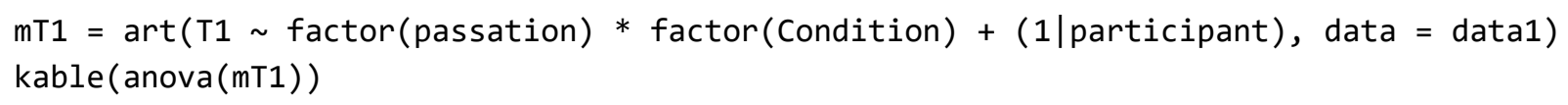

\begin{tabular}{llrrrr}
\hline factor(passation) & factor(passation) & 1.9240618 & 5 & 18 & 0.1400870 \\
\hline factor(Condition) & factor(Condition) & 0.4965391 & 2 & 36 & 0.6127404 \\
\hline factor(passation):factor(Condition) & factor(passation):factor(Condition) & 0.7280084 & 10 & 36 & 0.6932765
\end{tabular}

\subsubsection{Item T2}

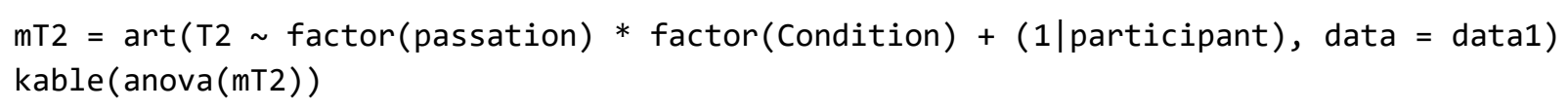

\subsubsection{Item T3}


mT3 = art $($ T3 factor (passation) $*$ factor(Condition $)+(1 \mid$ participant $)$, data $=$ data1 $)$

kable(anova(mT3))

Term

factor(passation)

factor(Condition)
F Df Df.res

$\operatorname{Pr}(>\mathrm{F})$

factor(passation)

factor(Condition)

factor(passation):factor(Condition) factor(passation):factor(Condition)

$\begin{array}{rrrr}3.6284144 & 5 & 18 & 0.0191057 \\ 14.1288592 & 2 & 36 & 0.0000296 \\ 0.8916989 & 10 & 36 & 0.5499018\end{array}$

\subsubsection{Post-hoc pairwise test for T3}

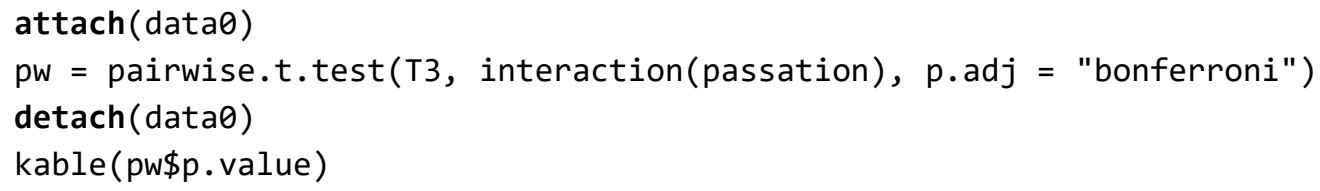

\begin{tabular}{rrrrrr} 
& $\mathbf{1}$ & $\mathbf{2}$ & $\mathbf{3}$ & $\mathbf{4}$ & $\mathbf{5}$ \\
\hline 2 & 1.0000000 & NA & NA & NA & NA \\
\hline 3 & 0.3835758 & 1.0000000 & NA & NA & NA \\
\hline 4 & 1.0000000 & 1.0000000 & 0.9925147 & NA & NA \\
\hline 5 & 1.0000000 & 1.0000000 & 0.1501001 & 1 & NA \\
\hline 6 & 1.0000000 & 0.1654441 & 0.0107491 & 1 & 1
\end{tabular}

\section{5 .5 Item T4}

mT4 $=\operatorname{art}($ T4 $\sim$ factor (passation $) *$ factor (Condition $)+(1 \mid$ participant $)$, data $=$ data 1$)$ kable (anova (mT4))

\section{Term}

factor(passation)

factor(Condition)

factor(passation):factor(Condition) factor(passation):factor(Condition)

factor(passation)
F Df Df.res

$\operatorname{Pr}(>\mathrm{F})$

$\begin{array}{llll}1.3759234 & 5 & 18 & 0.2795487\end{array}$

$\begin{array}{llll}6.9665199 & 2 & 36 & 0.0027699\end{array}$

$0.4481359 \quad 10 \quad 36 \quad 0.9117118$

\subsection{ANOVA on ART for NASA TLX}

\subsubsection{Item Mental Load}

mTLXMental $=\operatorname{art}($ TLX1 $\sim$ factor $($ passation $) *$ factor $($ Condition $)+(1 \mid$ participant $)$, data $=$ data 1) kable(anova (mTLXMental))

Term 


\subsubsection{Item Physical Load}

mTLXPhysical $=\operatorname{art}($ TLX2 $\sim$ factor $($ passation $) *$ factor $($ Condition $)+(1 \mid$ participant $)$, data $=$ dat a1)

kable(anova(mTLXPhysical))

Term

F Df Df.res

factor(passation)

factor(Condition)

factor(Condition)

factor(passation):factor(Condition)

\begin{tabular}{rrrr}
0.5801659 & 5 & 18 & 0.7147635 \\
\hline 0.2068777 & 2 & 36 & 0.8140790 \\
\hline 1.4490538 & 10 & 36 & 0.1991451
\end{tabular}

\subsubsection{Item Temporal Load}

mTLXTemporal $=\operatorname{art}(\operatorname{TLX} 3 \sim$ factor $($ passation $) *$ factor $($ Condition $)+(1 \mid$ participant $)$, data = dat a1)

$\operatorname{kable}(\operatorname{anova}(\mathrm{m01}))$

Term F Df Df.res $\operatorname{Pr}(>\mathrm{F})$

\begin{tabular}{llrrrr}
\hline factor(passation) & factor(passation) & 2.055169 & 5 & 18 & 0.1189762 \\
\hline factor(Condition) & factor(Condition) & 5.638673 & 2 & 36 & 0.0074078 \\
\hline factor(passation):factor(Condition) & factor(passation):factor(Condition) & 1.000878 & 10 & 36 & 0.4610882
\end{tabular}

\subsubsection{Item Performance}

mTLXPerformance $=\operatorname{art}($ TLX4 $\sim$ factor $($ passation $) *$ factor $($ Condition $)+(1 \mid$ participant $)$, data $=$ data1)

kable(anova(mTLXPerformance))

Term

factor(passation)

factor(Condition)

factor(passation):factor(Condition) factor(passation):factor(Condition)

factor(passation)
F Df Df.res

$\operatorname{Pr}(>\mathrm{F})$

$0.4501301 \quad 5 \quad 18 \quad 0.8076809$

$\begin{array}{llll}5.6579343 & 2 & 36 & 0.0073000\end{array}$

1.410111110

\subsubsection{Item Effort}

mTLXEffort $=\operatorname{art}($ TLX5 $\sim$ factor $($ passation $) *$ factor $($ Condition $)+(1 \mid$ participant $)$, data $=$ data 1) kable (anova(mTLXEffort)) 


\begin{tabular}{llrrrr}
\hline factor(passation) & factor(passation) & 0.6042859 & 5 & 18 & 0.6975429 \\
\hline factor(Condition) & factor(Condition) & 6.2034512 & 2 & 36 & 0.0048430 \\
\hline factor(passation):factor(Condition) & factor(passation):factor(Condition) & 1.6135575 & 10 & 36 & 0.1422056
\end{tabular}

\subsubsection{Item Frustration}

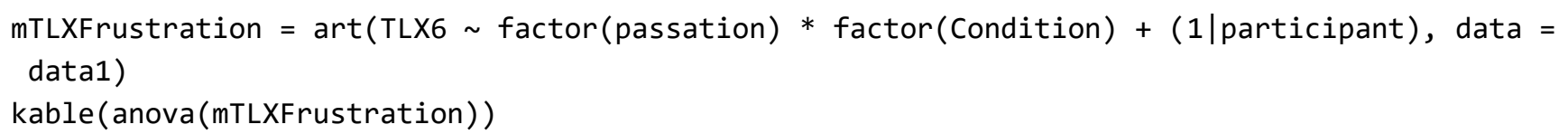

Term F Df Df.res $\operatorname{Pr}(>\mathrm{F})$

\begin{tabular}{llrrrr}
\hline factor(passation) & factor(passation) & 2.0113372 & 5 & 18 & 0.1256366 \\
\hline factor(Condition) & factor(Condition) & 7.2227285 & 2 & 36 & 0.0023049 \\
\hline factor(passation):factor(Condition) & factor(passation):factor(Condition) & 0.9659337 & 10 & 36 & 0.4886218
\end{tabular}

